Influence du discours : parole légitimée, engagée et persuasive. Analyse des discours de la concorde civile du Président

\title{
Abdelaziz Bouteflika
}

\section{Ouahiba BENBAKKAR}

\section{Université Alger 2}

\section{Résumé :}

Le discours politique fait partie intégrante des études en analyse du discours, notamment celles qui s'intéressent à l'examen des stratégies discursives visant à influencer autrui. L'auteure analyse la cohérence thématique des discours de la concorde civile du Président Bouteflika. Elle tente de développer une approche dynamique dans laquelle elle distingue différents types de progression thématique. En outre, elle soutient l'idée, inspirée de certaines études en linguistique textuelle, que le rôle des stratégies discursives est d'assurer l'influence de l'instance politique, à travers son discours, lui permettant ainsi d'exercer une action, un pouvoir sur l'instance citoyenne.

\section{الملخص:}

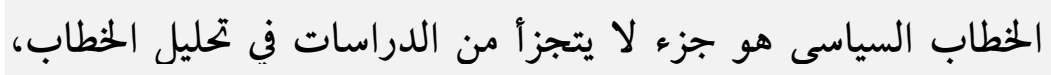

وبخاصة تلك التى تدرس الاستراتيجيات الخطابية الهادفة للتأثير على الآخر.

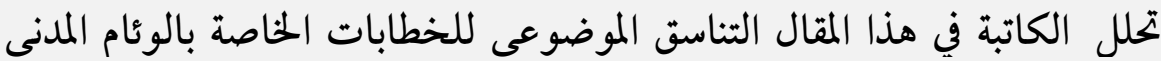
للرئيس عبد العزيز بوتفليقة. و هى بذلك تحاول وضم نهج ديناميكى الذي يميز

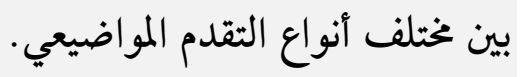
بالإضافة إلى ذلك، تدعم الكاتبة الفكرة، التى تستوحيها من بعض الدراسات في

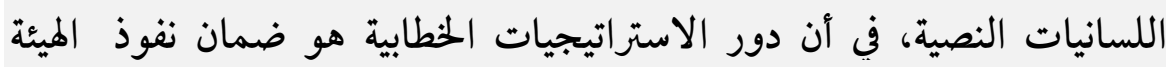
السياسية والسماح لها بممارسة سلطتها على الهيئة المواطنية. 


\section{Introduction :}

«Toute parole est tentative d'influence d'autrui ${ }^{(1)}$.

Les discours de la concorde civile du Président de la République algérienne instaurent une relation entre deux instances : l'orateur (le Président) et l'auditoire (le peuple algérien). Ces discours mettent en évidence différentes stratégies discursives qui visent à faire adhérer l'auditoire et à l'orienter de façon à construire un pathos déterminé convergeant avec l'intentionnalité argumentative de l'émetteur.

En fait, on ne saurait parler de l'argumentation sans évoquer nécessairement le concept d'influence qui serait sous-jacent à toute tentative d'exercice de pouvoir sur autrui. C'est pourquoi les recherches en analyse du discours ne cherchent en aucune façon à démontrer le degré de vérité des arguments mais leur degré d'influence, c'est-à-dire le faire croire, le faire faire par le biais du langage. Autrement dit, elles cherchent à mettre à jour la force du langage à faire adhérer l'autre au point de vue défendu par l'énonciateur.

A cet effet, il serait très important de signaler que l'influence ne se s'impose pas uniquement par la force des idées mais aussi et surtout par l'ensemble des valeurs sociales et culturelles perçues comme vraies par telle ou telle société et qui ne peuvent pas, par contre, être vérifiées. Cela nous conduit à penser que tout discours dépend du contrat de communication entre les instances énonciatives 
qu'il lie. Dans cette contribution, nous allons tenter de mettre en évidence quelques aspects par lesquels s'affirme l'influence de la parole présidentielle en nous attachant plus particulièrement à montrer comment les stratégies discursives déployées sont manipulées en vue d'atteindre des finalités précises. Notre attention sera portée également, en nous adossant aux apports de la linguistique textuelle, aux différents types de progression thématique qui véhiculent exhibent et dramatisent l'engagement, la persuasion et la légitimité du discours présidentiel.

\section{La progression thématique}

«Tout texte est pris dans une tension entre cohésion (liée à la structure thématique, à la connexion et à la concaténation des thèmes successifs) et progression ${ }^{(2)}$. La progression thématique des énoncés est aujourd'hui un point central dans les travaux d'analyse $\mathrm{du}$ discours car «elle rend compte des enchainements phrastiques d'un texte en explicitant sa cohésion et sa progression transphrastique ${ }^{(3)}$.

Cette progression est fondée sur la distinction entre les deux notions thème/rhème, elle est basée sur «la vieille question de l'ordre des mots dans la phrase posée » ${ }^{(4)}$. L'ordre des mots dans une phrase montre la différence entre les deux concepts : ainsi, «une partie d'un groupe nominal ou verbal, ou d'une phrase, peut avoir de façon indépendante du découpage en constituants syntaxiques une valeur de thème ou de rhème, liée à sa place dans la dynamique 
de la phrase et à sa visée communicative : information présentée comme (thème) ou comme nouvelle (rhème) $\gg{ }^{(5)}$. Les travaux qui ont été réalisés récemment sur la question de la progression thématique des énoncés mettent en évidence la fonction cohésive ${ }^{(6)}$.

Des différentes reprises thématiques et le rôle du rhème dans la progression thématique.

Les deux concepts de thème et de rhème permettent d'apporter une fine description de la structure de la phrase mais aussi une étude permettant de vérifier «les mouvements textuels de reprise et de progression de l'information ${ }^{(7)}$.

C'est pourquoi il nous semble très important de montrer la différence entre thème et rhème avant de passer aux trois types de progression proposés par le linguiste Adam.

\subsection{Thème / rhème}

La distinction entre ces deux concepts est traitée dans le dictionnaire d'analyse du discours. Elle « est en effet centrée d'une part, sur le degré d'informativité et de dynamique communicative à l'intérieur d'une phrase et, d'autre part sur la grammaire des enchainements phrastiques » ${ }^{(8)}$.

C'est grâce à la progression de l'information que nous arrivons à comprendre le fonctionnement de la phrase où chaque unité occupe une place ; ainsi, " la place de début (thème) [...] et de fin de phrase (rhème) possèdent un degré différent de dynamisme 
communicatif du plus bas degré pour le Thème (ce dont on parle) au plus haut degré pour le Rhème (ce qu'on dit du thème) 》" ${ }^{(9)}$.

Le linguiste Ducrot, considère que l'organisation sémantique est examinée sur cinq points, dont le troisième traite la distinction entre les deux concepts thème et rhème appelés aussi thème et propos. Cette distinction est d'ordre psychologique ; pour lui ${ }^{(10)}$.

«le thème [...] d'un énoncé, c'est ce dont parle le locuteur» il ajoute ${ }^{(11)}$. qu'il est «le sujet psychologique » de l'énoncé.

Le propos ou le rhème est défini comme «l'information qu'il entend apporter relativement à ce thème » qu'il appelle aussi «le prédicat psychologique logique » ${ }^{(12)}$.

Dans cette définition, le thème est considéré comme «un objet extérieur auquel l'énoncé fait allusion ». Cela veut dire que le thème n'est pas forcément explicite par un énoncé mais il peut le contenir implicitement. En revanche, le thème peut être parfois et dans un énoncé explicite de par l'énoncé lui-même et ceci se fait par des marques linguistiques permettant de distinguer entre le thème et le propos.

Adam (2005) traite la question de la distinction entre les deux concepts thème/rhème, la phrase s'organise pour lui sur deux plans, «le groupe le plus à gauche, le thème, est du point de vue de l'énonciateur, le point de départ de l'énoncé » ${ }^{(13)}$. 
Il ajoute que le thème est «moins informant, en raison de son inscription dans le co-texte d'une reprise [...] ou en raison de son inscription dans le contexte d'un repérage déictique lié à la situation d'énonciation ». En effet, le thème est absent du texte, il se trouve dans le contexte de l'énonciateur et «la partie thématique est donc co(n) textuellement déductible » ${ }^{(14)}$.

Toujours dans la théorie du linguiste Adam, «le groupe le plus à droite, le rhème, correspond à ce qui est dit du thème, c'est l'élément phrastique posé comme le plus informant, celui qui fait avancer la communication ».

Récapitulons ce qui a été dit, le thème occupe la place à gauche de l'énoncé, le rhème occupe la place à droite. Parfois, le premier n'est pas forcément présent dans le texte, mais il peut être déduit du contexte, le second est présent dans le texte et il représente ce qui est dit du thème.

Ainsi dans l'énoncé suivant, le groupe de mots clivé opère une thématisation de la paix et une rhématisation de la réalisation de la réconciliation de l'Algérie avec elle-même.

$\left(E_{1}\right)$ : «C'est cette paix qui réalise la réconciliation de l'Algérie avec elle-même, c'est cette paix pour laquelle je me suis engagé avec vous ».

Aussi dans l'énoncé $\left(\mathbf{E}_{2}\right)$, le groupe de mots clivé met l'accent sur les bases de la stratégie, et de cette façon opère une thématisation du thème « base » : 
$\left(E_{2}\right)$ : « C'est sur toutes ces bases que j’ai fondé une partie de la stratégie de sortie de crise, dont le rétablissement de la paix est la condition nécessaire première ".

Mais «en assignant à ce concept une place dans la dynamique textuelle on dépasse la division de la phrase en thème (Th) et rhème (Rh) pour insister sur le point de départ (Th) de chaque nouvel énoncé », cela veut dire qu' " une phrase en amène une autre, elle la déclenche ; et le point d'aboutissement d'une phrase est très souvent la notion initiale de la phrase suivante » ${ }^{(15)}$.

Dans l'énoncé suivant $\left(\mathbf{E}_{3}\right)$, le thème « la paix » a eu pour rhème « la croissance économique » devenu à son tour le thème de la proposition qui la succède :

$\left(\mathbf{E}_{3}\right)$ : « Par ailleurs, la paix conditionne la croissance économique. Sans croissance économique, la paix restera fragile et menacée par d'autres formes d'expression de la violence sociale $»$.

\subsection{Les types de progression thématique}

Dans sa théorie basée sur la linguistique textuelle, Adam distingue trois grands types de progression thématique. Ces progressions sont «la plupart du temps mêlées à l'intérieur d'un même texte, et peuvent être partagées » ${ }^{(16)}$.

\subsubsection{La progression à thème constant}


Dans ce premier type de progression thématique « un même thème est repris à l'autre et associé à des rhèmes différents » ${ }^{(17)}$.

Cette reprise du rhème est souvent présente dans les séquences narratives, où elle apparait sous forme pronominale tout en ayant une relation avec d'autres rhèmes successifs.

La victoire peut être définie ainsi :

Schéma 1 :

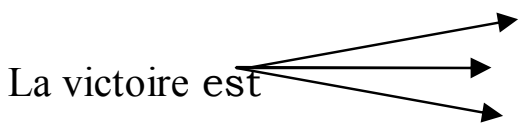

Le retour définitif à la paix

La reprise de la croissance économique

Le recul du chômage

La progression thématique dans l'énoncé suivant peut se résumer dans le schéma 02.

$\left(E_{4}\right)$ : «Car la victoire finale signifie le retour définitif de la paix dans notre pays, elle signifie la reprise de la croissance économique, le recul du chômage et le bien être social»

\section{Schéma 02:}

Cette relation ternaire entre le thème «victoire » et les trois rhèmes attire l'attention de l'auditoire qui voit en cette victoire le retour à la vie en paix. 


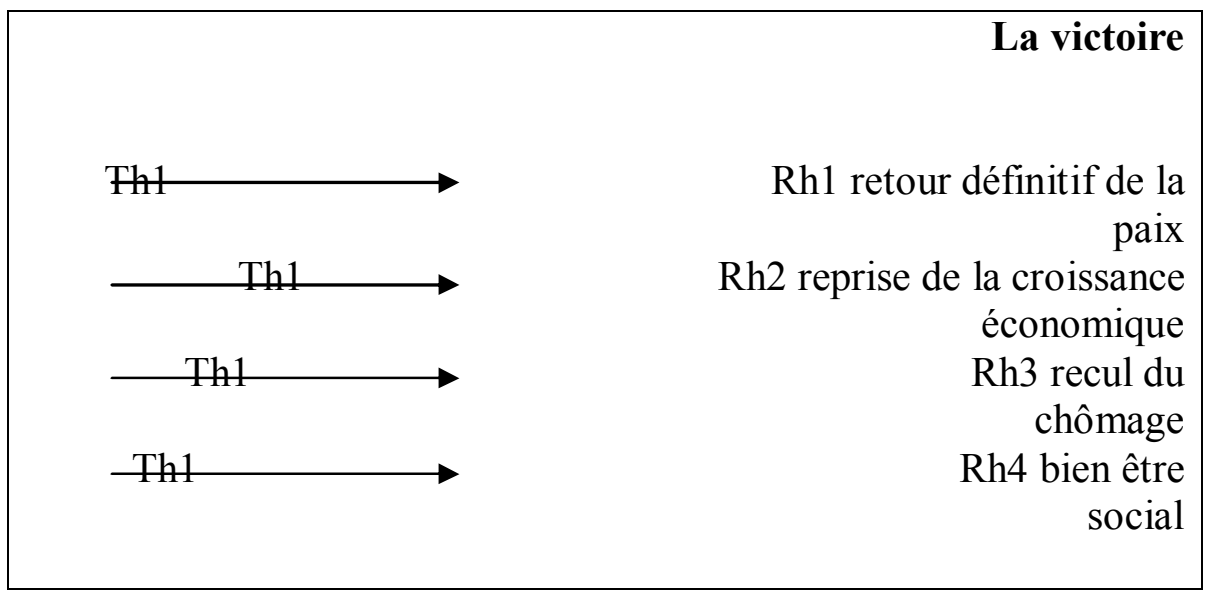

\subsubsection{La progression par thématisation linéaire :}

Adam définit ce type de progression thématique ainsi : «le $R h$ d'une première phrase devient le Th de la seconde dont le Rh fournit à son tour le Th de la suivante » ${ }^{(18)}$.

Dans l'énoncé suivant, «la concorde » est le thème de la proposition [P1], ce thème est explicité par le rhème « comportement », ce dernier à son tour est devenu par la suite le thème de la proposition $[\mathrm{P} 2]$ et a eu pour rhème « signification ».

(E) : «Que tout le monde sache que la concorde est un comportement [P1], un comportement dont la signification n'est connue que de ceux qui en ont été privés [P2] ». 
Schématisons cette analyse :

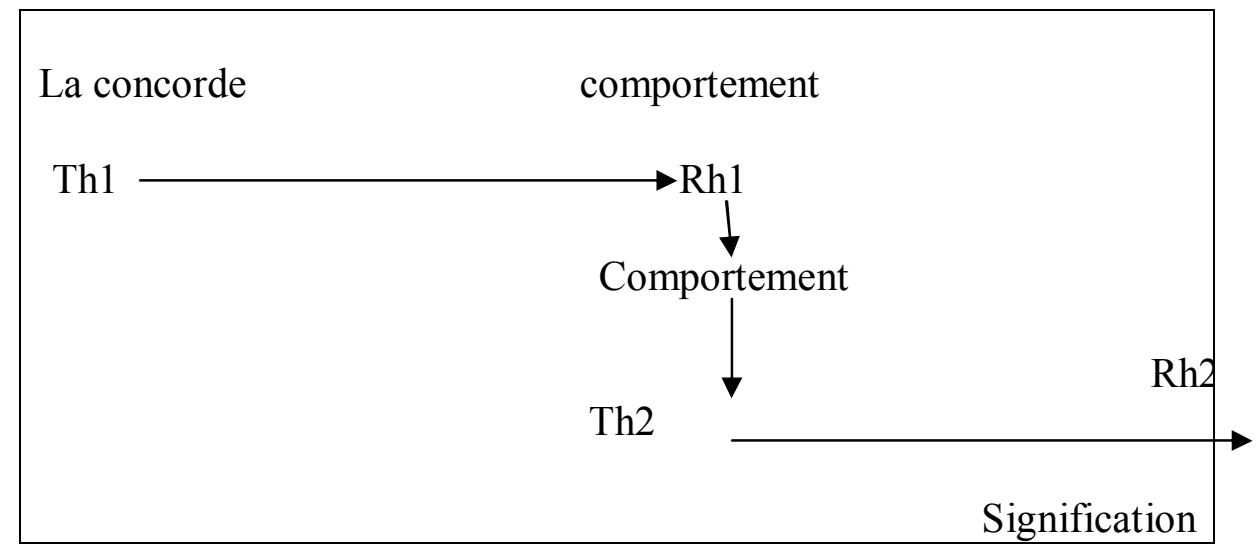

\subsubsection{La progression thématique combinée}

C'est une sorte de combinaison entre les deux modèles précédents. Cette progression est «une version avec redondance $d u$ thème initial Th1 et reprise du rhème Rh2 par deux fois » ${ }^{(19)}$.

Dans le même énoncé traite dans le paragraphe précédant, le thème de « la concorde civile» a eu un rhème «comportement», pour enfin arriver au rhème « signification » qui est l'aboutissement. 
Schématisons :

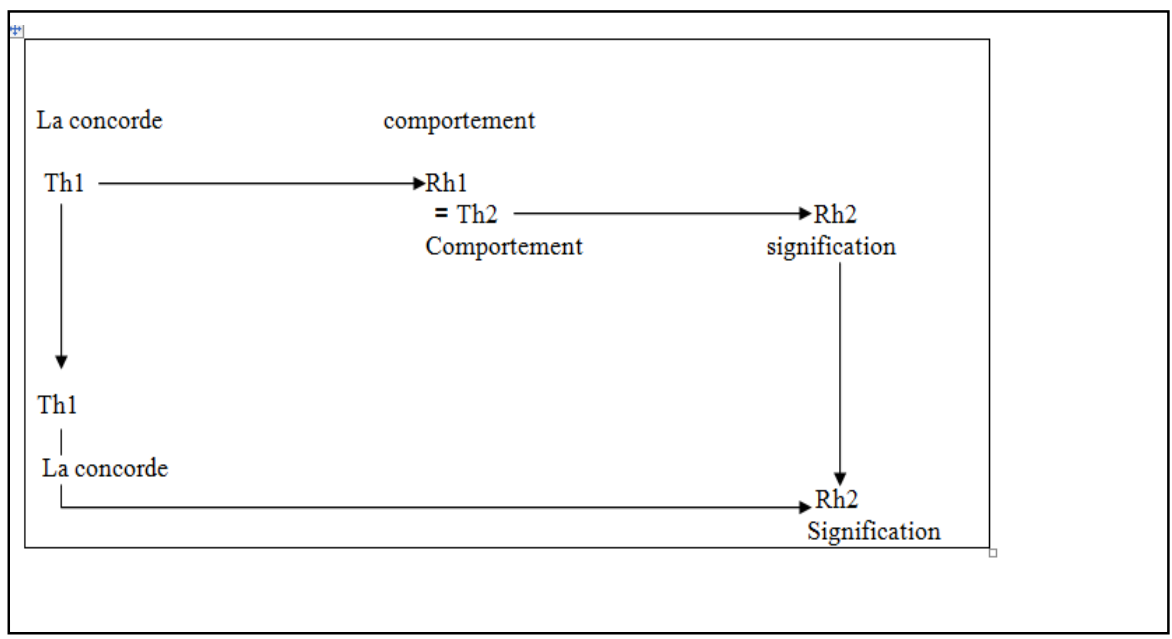

Ou encore :

La concorde

(Solution pour le

retour à la paix) signification (pour ceux qui ont connu le terrorisme)

\section{Cohérence thématique}

«Quand on parle, on fait allusion à un monde ("réel » ou « fictif »[...]), on construit une représentation : c'est la fonction descriptive de la langue. Mais on parle souvent en cherchant à faire partager à un interlocuteur des opinions ou des représentations relatives à un thème donné $\gg{ }^{(20)}$. 
A partir de ce postulat, nous procéderons à l'analyse des thèmes traités dans les discours de la concorde civile. Il est évident que tout discours dépend du contrat de communication entre les instances énonciatives qu'il lie. Mais il faut, pour la production d'un discours argumentatif, que ses instances soient pourvues d'un code commun, et qu'elles soient informées sur le contexte du discours en question.

Le discours de la concorde du Président de la République algérienne instaure une relation entre deux instances : l'orateur (le Président Bouteflika) et l'auditoire (le peuple algérien). Pour qu'il y ait une compréhension du message lancé par l'orateur, les deux instances doivent partager le même code et les mêmes connaissances du monde, ce qui est le cas pour nous.

Pour faire adhérer son auditoire à son discours, le Président doit construire son discours de façon qui lui permet d'aboutir à cela (agir sur l'opinion publique).

Pour analyser cette logique, nous allons examiner les différents thèmes présents dans le discours.

\subsection{La parole légitimée}

Le politique est un domaine où se jouent des rapports de force symboliques pour la conquête et la gestion d'un pouvoir qui ne peut s'exercer que dès lors qu'il se fonde sur une légitimité acquise et attribuée. Mais cela ne suffit pas, car le sujet politique qui est en 
quête de légitimité doit aussi se montrer crédible et persuader le plus grand nombre d'individus qu'ils doivent partager certaines valeurs.

Caractériser le discours de la concorde civile du point de vue de l'analyse du discours, c'est en premier lieu décrire le contrat de communication politique ainsi que l'identité de l'acteur de la scène politique le Président Bouteflika et en particulier les différentes formes de légitimité qui peuvent être attribuées à cette instance politique.

Dans le sens courant, la notion de légitimité est «un état de droit qui caractérise une personne au regard de sa situation (légitimité d'une union), d'une filiation (légitimité monarchique), d'un pouvoir conféré (légitimité démocratique) » ${ }^{(21)}$.

En analyse du discours, «la notion de légitimation peut être utilisée pour signifier que le sujet parlant entre dans un processus de discours qui doit aboutir à ce qu'on lui reconnaisse un droit à la parole et une légitimité pour dire ce qu'il dit» ${ }^{(22)}$.

Cela veut dire que la légitimation est un processus par lequel l'orateur se fait un droit à la parole pour dire son discours.

La légitimation accordée à un locuteur "peut lui venir soit d'une situation de fait [...] soit de la place qui lui est accordée par une institution quelconque comme lorsqu' [...] une personnalité politique fait une déclaration à la télévision $»{ }^{(23)}$. 
La place accordée à un Président lui donne le droit ou la légitimité de prendre la parole en public, de se construire une position de légitimité.

\section{$\left(\mathbf{E}_{6}\right):$ « Chers compatriotes »}

Cette séquence du discours du 02 août 1999 a une valeur inaugurale parce qu'elle ouvre le discours. L'orateur l'a commencé d'abord par l'adjectif «chers » introduisant cette formule de politesse et ayant un sens d'amitié et de familiarité du Président avec son peuple, ensuite le nom «compatriotes » lancé par l'orateur dans le but de créer une certaine relation avec son auditoire, une relation solide déjà existante justifiée par le fait que les deux instances énonciatives appartiennent au même pays qui est l'Algérie.

M. le Président Bouteflika, en commençant son discours par cette expression, se donne le droit, voire la légitimité, de prendre la parole devant un public cherchant des solutions à un problème qui a duré toute une décennie.

L'homme politique doit chercher quel peut être le plus grand dénominateur commun des idées du groupe auquel il s'adresse, tout en s'interrogeant sur la façon de les présenter. Il doit se soucier de la façon dont il présente ses arguments. Mais avant cela, il doit s'occuper d'abord de la façon de sa prise de parole. 


\section{$\left(E_{7}\right):$ « Chères sœurs chères frères ".}

En commençant le discours du 02 août 1999, avec ce fragment l'orateur souligne le type de relation qu'il veut établir avec son auditoire. D'abord, en employant les adjectifs «chères » et « chers » s'adressant aux Algériennes et aux Algériens, dans le but de créer cette intimité avec son public qui est créée ensuite par les deux noms « sœurs » et « frères » qui dépassent même l'amitié pour aller jusqu'à la familiarité.

\subsection{La parole engagée}

Dans le dictionnaire Larousse, le verbe pronominal « s'engager » a pris pour sens celui d' " exprimer publiquement par ses actes ou ses paroles une prise de position sur les problèmes sociaux, politiques ».

L'examen de la stratégie discursive de l'engagement, dans fragment suivant, est assuré par l'emploi du verbe «s'engager», traduit un certain état psychologique, et, employé à la première personne ce qui constitue le cas présent, a pour fonction de donner l'appréciation du locuteur sur la valeur de vérité de la proposition subordonnée.

$\left(E_{8}\right)$ : « C'est cette paix qui réalise la réconciliation de l'Algérie avec elle-même, c'est cette paix pour laquelle je me suis engagé avec vous $\gg$. 
Nous soulignons dans le fragment précédant l'engagement du Président pour instaurer la paix au sein de la société. Ce thème forme la cohérence thématique entre les deux discours mis en question. Le Président vise les thèmes suivants :

(i) Mettre fin aux actes des terroristes et par conséquent instaurer la paix.

(ii) Mettre en relation la question de la paix sociale et celle du développement économique de l'Algérie.

Le verbe lui-même «s'engager » conjugué au passé composé met l'accent sur l'engagement du chef de l'Etat pour une réconciliation du peuple algérien avec les auteurs des actes terroristes. Ici, le Président parle expressément de la «paix » et surtout avec une forte implication de sa personne et celle de son auditoire.

Cette implication de l'orateur se fait à travers l'utilisation du pronom personnel « je », c'est par ce « je » que le Président montre son engagement pour le projet de la concorde civile.

$\left(E_{10}\right)$ : « L'engagement du peuple pour la concorde civile oblige tous les agents de l'Etat à faire preuve de diligence dans le règlement des dossiers et d'un comportement responsable dans leurs relations avec les uns et les autres... ». 
Aussi dans l'énoncé $\left(\mathbf{E}_{\mathbf{1 1}}\right)$, lançant explicitement et expressément son «engagement» pour le projet en question, l'orateur vise à faire engager l'auditoire à son tour pour la loi de la paix.

$\left(E_{11}\right)$ : « Je me suis complètement investi dans cette entreprise nationale. J'ai pris, avec une rapidité déconcertante la responsabilité sur moi de donner une assise juridique à cette dynamique de paix et d'espoir pour tous $\gg$.

L'orateur montre avec plus d'expressivité son engagement pour ce projet, et ce, avec un « je », à travers lequel il met l'accent sur son investissement pour la loi de la paix.

Aussi, en lançant le lexème «responsabilité », qui est lourd de par son sens et de par sa présence dans cet environnement linguistique, l'orateur se porte responsable du projet de la concorde civile en lui donnant une assise juridique et surtout en la considérant comme allant de soi grâce aux marqueurs de personne «je » et « moi » représentant le point de vue «je suis responsable du projet de la concorde civile» comme assumé par l'orateur qui est le locuteur-origine de l'énoncé et comme préexistant à l'énonciation, ce qui nous fait considérer ce point de vue comme «allant de soi » ${ }^{(24)}$.

$\left(E_{12}\right)$ : «Tels sont les fronts sur lesquels je me bats et que j’essaye de maintenir en parfaite synergie ». 
L'orateur dans l'énoncé $\left(\mathbf{E}_{\mathbf{1 2}}\right)$ montre son total engagement pour les fronts pour lesquels il se bat, il le prouve par le déictique de personne « je ». L'emploi de ce « je » souligne l'engagement du Chef de l'Etat pour la maintenance des différentes bases de son projet concourant a un effet unique et avec une économie de moyen, ce que l'orateur résume en employant le lexème de «synergie ».

A travers ces discours, M. le Président annonce son engagement pour le projet et la loi de la concorde civile, mais comment va-t-il faire adhérer son auditoire à ce projet ?

Comment et avec quels procédés linguistiques réussira-t-il a persuader son public à dire « oui » à cette loi ?

\subsection{La parole persuasive}

La persuasion est vue par Chabrol comme « le produit des processus généraux d'influence » ${ }^{(25)}$.

En vue d'influencer et de persuader, l'orateur utilise un processus par lequel il provoque «l'adhésion des esprits aux thèses qu' [il] présente à leur assentiment » ${ }^{(26)}$.écrit ce que confirme Perlman, il est possible «d'appeler persuasive, une argumentation qui ne prétend valoir que pour un auditoire particulier et d'appeler convaincante celle qui est censée obtenir l'adhésion de tout être de raison $\gg{ }^{(27)}$. 


\subsubsection{La dramatisation}

$\left(E_{13}\right)$ : « J'avais perçu, lors de la compagne électorale votre appel pour que cesse à jamais la tragédie fratricide qui endeuille le peuple algérien, depuis prés d'une décennie »

Dans cette séquence, l'autorité suprême cherche à rendre plus dramatique l'état de paix dans la société algérienne, et ce, par des moyens linguistiques : «tragédie fratricide ».

Le lexème «tragédie » est lui-même significatif et a le même sens que celui de « drame ».

L'orateur vise à rendre les citoyens plus sensibles à la question de la paix en Algérie, en faisant de cette situation un événement funeste, tragique, bref dramatique.

Aussi par l'adjectif « fratricide », le Président cherche à créer une certaine réaction humaine de la part de son auditoire. En ajoutant le mot « fratricide» à « tragédie », le Chef de l'Etat met l'accent sur cette situation sociale opposant des personnes qui doivent être solidaires, solidaires au sein d'une société à laquelle ils appartiennent.

Dans la suite de cet énoncé, le verbe «endeuille» qui englobe dans son sens la mort provoque un sentiment de tristesse dans les esprits de ceux qui l'écoutent et les plonge dans un sentiment de « deuil ». 
En créant ce sentiment de tristesse, de deuil, de déchirement, l'orateur rend son auditoire plus sensible à la question de la paix, et par conséquent il arrive a saisir le pathos .

Il réussit donc intelligemment à faire adhérer son public a son message, et ce, en se montrant comme le sauveur, celui qui apporte la solution au problème, un problème qui concerne des vies humaines et qui a dure «près d'une décennie » de mort de viole, etc.

L'orateur par l'image qu'il se donne arrive à affecter son autorité et à créer une réaction positive par rapport a ce qu'il propose, « le projet de la concorde civile » qui est pour lui la solution a deux problèmes : le rétablissement de la situation sécuritaire en Algérie et la sortie de la crise économique.

$\left(E_{14}\right)$ : «Le processus que j'ai enclenché est parti d'une situation de fait tout à fait aléatoire pour déboucher sur une cessation définitive de la violence, mettre un terme à la «Fitna » et éteindre l'incendie qui a ravagé le pays et les hommes »

La question de la paix dans cette séquence est thématisée différemment. Ciblant son public, le Président utilise d'abord la notion de «Fitna », ce terme appartient à la langue arabe mais aussi à la religion musulmane ${ }^{(28)}$, un terme qui signifie «le chantage social » causé par les groupes armés. 
Ensuite, à travers l'emploi le mot « incendie », l'énonciateur fait appel à la stratégie de la dramatisation de la situation sécuritaire en vue de persuader son public.

M. le Président utilise toutes ces appellations dans le but de changer l'opinion de son public en rendant la situation encore plus dramatique qu'elle ne l'est. Ce qui lui permet de sensibiliser le peuple vis-à-vis du devenir sécuritaire.

Le verbe «ravager», met l'accent sur les dommages considérables causés par la violence des groupes armés. Ainsi, l'orateur se représente comme celui qui tire le pays et les hommes de ce grave problème. A travers l'ethos, l'énonciateur crée une réaction humaine chez son auditoire. En créant ce pathos, il est arrive à persuader son public.

\subsubsection{La manipulation}

$\left(E_{15}\right)$ : «Chacun a pu constater que depuis le 15 avril 1999, l'image de l'Algérie à l'extérieur, a été positivement et significativement modifiée. Notre pays reprend de la considération, son audience se rétablit graduellement, il est devenu un partenaire politique dont l'avis est recherché par les uns et les autres $»$.

En faisant appel à la date de son élection comme Président de la République algérienne, M. Bouteflika met l'accent sur les changements depuis le début de son règne. Depuis le 15 avril 1999 
(une date qui est antérieure par rapport au jour du lancement du discours), l'Algérie a remis en cause son image à l'extérieur. S'intéressant à l'image de son pays, le Chef de l'Etat fait appel à cette réussite qui est «considérable » pour lui tout comme pour le peuple algérien, un peuple qui a perdu la confiance et les relations qu'il entretenait avec l'extérieur, et ce depuis le déclenchement de la guerre civile à l'intérieur de la patrie.

L'orateur tente d'influencer son public en lui parlant de sa réussite, une réussite qui rappelle une autre, celle du rétablissement de la situation sécuritaire en Algérie.

Aussi dans l'énoncé suivant, la manipulation est flagrante. L'orateur vise à persuader son auditoire pour ce qu'il appelle « la paix des hommes » et a le manipuler contre ce qu'il appelle « la paix des armes », celle-ci est pour lui destructive puisqu'elle conduit a «plus de morts et plus de misère », celle-là est constructive comme elle conduit a « moins de misère ».

$\left(E_{16}\right)$ : « il y a certes la paix des armes dont la logique conduit à plus de destruction, plus de morts, plus de misère. Son avènement restera toujours aléatoire. Il y a la paix des hommes, la paix des cours, la paix des esprits qui, dans tous les cas, conduit immédiatement à plus de fraternité, plus de solidarité, plus de construction, et moins de misère » 
Pour notre Président, il est donc plus raisonnable d'aller chercher la paix par la paix et non par les armes à l'intérieur de la patrie « l’Algérie ».

\subsubsection{La compassion}

La compassion est l'une des stratégies sur lesquelles s'appuie la persuasion dans le discours de la concorde civile. Elle est définie dans le dictionnaire « Larousse » comme «le sentiment qui pousse à partager les souffrances d'autrui ».

$\left(E_{17}\right)$ : "Chaque Algérienne et chaque Algérien, qui adhère à cette démarche, a le devoir de répandre autour de lui, les graines de la solidarité et du respect vis-à-vis de tous les Algériens, mais plus particulièrement vis-à-vis des plus vulnérables, dans ce processus de paix, qui sont les victimes du terrorismes ou ceux qui, par les moments d'errements, ont répondu enfin, à l'appel de leur peuple et réintégré les rangs de la société. A ceux-ci, ne leur rappeler pas les conditions d'hier, aidez les à regarder vers l'avenir qu'ils ont choisi de bâtir avec vous. A ceux-là, compensez l'absence de l'être cher par votre présence solidaire $»$.

Dans cette séquence, l'orateur lance un appel compassionnel, à travers son discours. Cet appel répond a une intention de faire 
partager la partie des Algériens(*).qui ne sont pas des terroristes, et qui n'ont pas été touches par des actes du terrorisme. Le Chef de l'Etat demande à cette majorité du peuple algérien d'accepter, et avec un grand cœur, la réintégration des terroristes dans la société, et en même temps de consoler ceux qui ont perdu un «être cher» dit il. S'adressant au peuple algérien, à l'impératif, l'orateur à travers les verbes «aider» et «compenser» fait appel a cette stratégie de compassion dans le but de provoquer un sentiment de partage et de consolation chez l'auditoire.

Aussi par les lexèmes «solidarité » et « respect», $\mathrm{M}$ Bouteflika vise à instaurer certains comportements au sein du pays qu'il préside, un respect mutuel entre les citoyens, et une solidarité partagée entre les membres de la société algérienne.

C'est pourquoi nous pensons que l'orateur, à travers cette consolation, arrive à persuader bel et bien son auditoire à adhérer au projet de la concorde civile.

\subsubsection{La séduction}

La persuasion est fondée sur la dramatisation, la manipulation, la compassion mais aussi la séduction.

$\left(E_{18}\right)$ : «J'ai initié une loi dans ce cadre ; elle a été votée par le parlement, dans son écrasante majorité sans qu'aucune voix contre ne soit exprimée. Cela est déjà en soi un soutien politique

(*)Cette partie des Algériens est une majorité et a massivement adhéré à la loi de la concorde civile lors du référendum. 
d'une grande importance. Ma foi en la générosité, la mansuétude, le bon sens et la grandeur du peuple algérien s'en est trouvée plus que jamais renforcée ».

En utilisant la stratégie de la flatterie, le Président Bouteflika vante les qualités du peuple algérien. Un peuple généreux, qui est pour lui indulgent, ayant du bon sens et de la grandeur, toutes ces qualités sont vantées dans le but de satisfaire l'orgueil de l'auditoire.

Aussi dans l'énoncé $\left(\mathbf{E}_{\mathbf{1 4}}\right)$, l'orateur à travers le verbe "décider», montre que la décision finale est celle du peuple et qu'elle lui revient « souverainement», cet adverbe souligne la liberté et la démocratie en Algérie. Le peuple est donc libre de donner sa voix qu'elle soit négative ou positive pour le projet propose. Les Algériens sont invites à voter pour ou contre la loi de la concorde civile.

$\left(E_{19}\right)$ : «Ce n'est pas à une consultation ordinaire que le peuple algérien est convié aujourd'hui. Les solutions à la crise qui frappe notre pays existent et les conditions peuvent en être remplies, s’il le décide souverainement ».

Le peuple algérien est donc souverain, il a le libre choix pour le projet de la concorde civile. En utilisant la stratégie de la flatterie analysée dans les deux énoncés précédents, le Chef de l'Etat vise a travailler sur le pathos, dans la mesure où il a l'intention de séduire voire de persuader son auditoire, un auditoire qui est censé prendre la bonne décision pour le projet de la paix. 


\subsubsection{La répétition}

La répétition dans la langue est considérée par certains linguistes comme une stratégie de persuasion. Elle est définie par Breton $^{(29)}$.

comme «le phénomène d'abandon de la conscience critique, qui finit par se persuader ». Cela veut dire que l'auditoire finit par se persuader car « en quelque sorte, puisqu'il est répété, le message a déjà été justifié » ${ }^{(30)}$.

Le politicien fait appel à cet stratégie dans le but de fatiguer la conscience du public, pour qu'i adhère au projet de la concorde civile.

Le groupe de mot « assise juridique » a été répété maintes fois :

$\left(E_{20}\right)$ : «C'est aussi sur toutes ces bases que j'ai initié un processus de rétablissement de la concorde civile, en donnant une assise juridique à une situation de fait existante depuis $1997 »$.

$\left(E_{21}\right)$ : « Je me suis complètement investi dans cette entreprise nationale, j'ai pris avec une rapidité déconcertante la responsabilité sur moi de donner une assise juridique à cette dynamique de paix et d'espoir pour tous ».

$\left(E_{22}\right)$ : «L'assise juridique signifie clairement que l'autorité politique prend sur elle de conférer un cadre légal fixant clairement les conditions de prise en charge des rapports de fait, nés de l'attitude de certains groupes armés ...». 
«L'assise juridique » signifie les fondements et les bases juridiques sur lesquels s'appuie le projet de la concorde civile. Insistant sur cette assise, l'orateur fait appel à la loi de la concorde civile. Dans les trois énoncés $\left(\mathbf{E}_{15}\right),\left(\mathbf{E}_{16}\right),\left(\mathbf{E}_{17}\right)$, cités plus haut, la répétition accentuant les bases juridiques du projet de la concorde civile, crée comme le dit Breton ${ }^{(31)}$.

«un sentiment d'évidence» chez l'auditoire. Ce dernier prend le message comme déjà justifie, et par conséquent il y adhère sans aucune hésitation. Une adhésion due à une fatigue mentale puisque à force de lui répéter le message, l'auditoire finit par dire «oui » à ce que l'on présente. Aussi dans les énoncés suivants, le lexème « engagement » est répété par l'orateur dans son discours de la concorde civile :

$\left(E_{23}\right)$ : « J'ai donné ma parole pour l'Aman, et si cet engagement express est un des fondements de ma démarche, par votre vote massif, il devient aussi le vôtre ».

$\left(E_{24}\right)$ : « L'engagement du peuple pour la concorde civile oblige tous les agents de l'Etat à faire preuve de diligence dans le règlement des dossiers et d'un comportement responsable dans leurs relations avec les uns et les autres... ».

$\left(E_{25}\right)$ « L'engagement du peuple est, enfin, une source d'émulation supplémentaire pour ceux qui sont encore dans la voie de la violence, pour se ressaisir...». 
En répétant le mot « engagement », l'orateur met l'accent sur l'engagement pris par sa personne et par le peuple algérien lors du referendum pour la loi et le projet de la concorde civile. Cette répétition vise à instaurer un sentiment de responsabilité des Algériens vis-a-vis du projet et de sa continuité. Mais l'engagement n'est pas seulement de la part du peuple mais aussi de la part des agents de l'Etat et du Président lui-même.

Cette répétition bien faite par l'orateur nous conduit à dire que l'orateur réussit à faire adhérer l'auditoire au projet de la concorde civile.

\subsubsection{Les mots à forte connotation}

La connotation d'un mot apparait comme une valeur «ajoutée », qui «relève moins de la linguistique à proprement parler que de la stylistique, de la psycholinguistique ou de la sociolinguistique ${ }^{(32)}$.

Pour C. Kerbrat-Orecchioni ${ }^{(33)}$.

« la connotation d'un terme, c'est une partie seulement de sa signification [...] à savoir l'ensemble des valeurs qui viennent se surajouter aux traits dénotatifs, lesquels sont directement liés aux propriétés $d u$ référent discursif $\gg$. Cela veut dire que c'est le référent discursif qui donne ce sens dénotatif à un terme ayant déjà un sens dit « dénotatif ${ }^{(34)}$. 
Ce sens connotatif vient des représentations sociales partagées entre l'orateur et son public. L'orateur fait appel à ce genre de stratégie dans le but de rendre son auditoire apte à accréditer les thèses qu'il présente et qu'il défend.

Dans l'énoncé suivant, le lexème «paix» autour duquel s'articule tout le discours, présente une signification particulière et pour l'orateur et pour le peuple algérien :

$\left(E_{26}\right)$ : « c'est cette paix qui réalise la réconciliation de l'Algérie avec elle-même, c'est cette paix pour laquelle je me suis engagé avec vous $\gg$.

\section{Conclusion}

Il faut reconnaitre que le discours politique met davantage en œuvre une visée d'incitation à penser et à faire qu'une visée de démonstration. Il s'agit moins d'établir une vérité en raison, indépendamment des opinions, que de chercher à transformer (ou renforcer) des opinions empreintes d'émotion. La mise en scène du discours politique oscille entre l'ordre de la raison et l'ordre de la passion, mélangeant logos, ethos et pathos pour tenter de répondre à la question qu'est censé se poser le citoyen : «Qu'est-ce qui fait que je devrais adhérer à telle ou telle valeur ? » Pour l'homme politique, c'est une affaire de stratégie dans le choix et le mode de présentation des valeurs. 
Tout se mêle donc dans cette subjectivité ; il est cependant possible de distinguer dans la mise en scène du discours politique, les traits qui sont destinés à présenter une certaine image de soi (ethos) et ceux qui sont destinés à toucher le public par une mise en scène dramatisante (pathos).

Dans ses discours de la concorde civile, le Président Bouteflika met en place une image de soi, une image qui existe par son statut de Président. Ainsi, l'orateur se donne la légitimité de prendre la parole en public.

A travers des stratégies d'engagement et de persuasion, le Chef de l'Etat travaille sur le pathos dans le but d'influencer son public pour:

(i) Le projet et la loi de la concorde civile, visant à instaurer la paix au sein de la société, et a mettre fin a des actes de terrorisme qui ont dure une décennie en Algérie.

(ii) Montrer l'importance de la paix dans le développement économique du pays qui en est une condition nécessaire.

(iii) Inciter les citoyens à prendre en charge les terroristes et les victimes des actes de ceux qui ont levé leurs armes contre l'Etat. 


\section{Références bibliographiques :}

(1) Mucchielli, A. (2009), L'art d'influencer. Analyse des techniques de manipulation, Paris, Armand colin, p. 7.

(2) Adam, J-M. (2005), La linguistique textuelle. Introduction à l'analyse textuelle des discours, Paris, Armand Colin, p.47.

(3) Charaudeau, P., et, Maingueneau, D. (2002), Dictionnaire d'analyse du discours, Paris, Seuil, p. 573.

(4) Ibid., p. 47.

(5) Ibid., p. 45

(6) La fonction cohésive est prouvée à travers l'analyse des thèmes et des rhèmes.

(7) Ibid., p. 46.

(8) Charaudeau, P., et, Maingueneau, D. (2002), Dictionnaire d'analyse du discours, Paris, Seuil, p. 572.

(9) Ibid.

(10) Ducrot, O., et, Schaeffer, J-M. (1995), Nouveau dictionnaire encyclopédique des sciences du langage, Paris, Seuil, p, 541.

(11) Ibid. P, 542.

(12) Ibid.

(13) Adam, J-M. (2005), La linguistique textuelle. Introduction à l'analyse textuelle des discours, Paris, Armand Colin, p.45. 
(14)

Adam, J-M. (1999), Linguistique textuelle. Des genres de discours aux textes, Paris, Hachette, p. 135.

(15)

Adam, J-M. (2005), La linguistique textuelle. Introduction à l'analyse textuelle des discours, Paris, Armand Colin, p.47.

(16)

Adam, J-M. (1992), Les textes types et prototypes, Paris, Nathan, p. 17.

(17)

Charaudeau, P., et, Maingueneau, D. (2002), Dictionnaire d'analyse du discours, Paris, Seuil, p. 573.

(18) Adam, J-M. (2005), La linguistique textuelle. Introduction à l'analyse textuelle des discours, Paris, Armand Colin, p.45.

(19) Ibid.

(20) Adam, J-M. (1999), Linguistique textuelle. Des genres de discours aux textes, Paris, Hachette, p. 135.

(21) Charaudeau, P., et, Maingueneau, D. (2002), Dictionnaire d'analyse du discours, Paris, Seuil, p. 340.

(22) Ibid.

(23) Ibid.

(24) Le fait de considérer un énoncé comme « allant de soi » est introduit en analyse du discours par le linguiste Haillet, P, P, dans son ouvrage « pour une linguistique des représentations discursives » (2005, P. 63). Qui à son tour s'est inspiré de la théorie de Ducrot, O. 
(25)

Charaudeau, P., et, Maingueneau, D. (2002), Dictionnaire d'analyse du discours, Paris, Seuil, p. 428.

(26) Ibid.

(27) Ibid.

(28) Le peuple algérien a pour langue maternelle « la langue arabe » et pour religion « l'Islam »

(29) Breton, P. (2000), La parole manipulée, Paris, La découverte, p. 95.

(30) Breton, P. (2000), La parole manipulée, Paris, La découverte, p. 95.

(31) Ibid.

(32) Kerbrat-Orecchioni, C. (2002), L'Enonciation, de la subjectivité dans le langage, Paris, Armand Colin, p. 131.

(33) Ibid.

(34) Dénotatif ici est employé par opposition à connotatif. 